\title{
Reconstruction of Two-dimensional Image to Three-dimensional Point Cloud Data for Detection of Empty Rot Defects in Tree Growth
}

\author{
Shufeng Jiang ${ }^{1,2}$, Keqi Wang ${ }^{1, *}$ \\ ${ }^{I}$ College of Mechanical and Electrical Engineering, Northeast Forestry University, Harbin, \\ Heilongjiang, China \\ ${ }^{2}$ College of Mechanical and Electrical Engineering, Qiqihar University, Qiqihar, Heilongjiang, China \\ *Corresponding Author.
}

\begin{abstract}
In the application of nondestructive detecting of trees, it is a technical problem to use radar waves to detect tree specimens with growth defects, how to segment defect areas after obtaining two-dimensional images, and reverse simulate the detection results with three-dimensional point cloud data. Therefore, the method of extracting boundary information according to color features is studied to extract the boundary curve of empty rot area, and the selection of higher precision extraction algorithm is determined by comparing the boundary extraction results of HSV color space and RGB color space in laboratory According to the extracted void boundary curve, the reverse modeling is carried out, and the mapping from $2 D$ inspection gray image to $3 D$ space is realized, The point cloud data reconstruction needed for $3 D$ modeling of multi-curved surfaces is obtained in reverse. The boundary curve extraction algorithm in this study is used to process the images of nondestructive testing of trees. Through comparative experiments and error analysis, the accurate modeling conclusion from inversion of $2 D$ images to $3 D$ point cloud data reconstruction by radar wave detection is verified, and the Core issue problem of point cloud reconstruction in the ill-conditioned area of tree growth and decay detected by radar wave is solved.
\end{abstract}

Keywords: Nondestructive detecting of trees, Feature boundary extraction, Three-dimensional model of hollow area with trees, Point cloud data reconstruction

\section{Introduction}

With the development of artificial intelligence technology and forestry engineering automation technology, tree nondestructive detecting technology has developed from the initial ray detection to obtain information feedback in the form of two-dimensional pictures to the level of three-dimensional visual information feedback. So, whether the trees growth condition monitoring, and to detect the quality of wood processing production in advance, based on radar wave nondestructive testing technology is the modern trees effective engineering technology of efficient utilization of resources, and identification and the basic method of the inversion of the detected defects is obtained by using two-dimensional image defect area image segmentation. So, how to get tree growth defects by radar detection of three-dimensional point cloud data, or the detection of the growth condition of using artificial intelligence technology in the process of 3D simulation, and based on 3D visualization test results for internal corrosion degree of empty trunk data contrast, trees is the radar wave of the future development direction of nondestructive testing technology.

At present, the feedback data of tree radar wave detection is a color two-dimensional map. In the picture, only the irregular curved boundary of the birth area of sky decay can be simply presented, or the edge of the white and white color strip with no clear boundary. Moreover, it is impossible to obtain the three-dimensional shape and degree of decay of the specific growing hollow rotten areas inside the trunk, so it requires professional personnel to conduct secondary analysis before the detection data can be consistent with human three-dimensional vision. At the same time, the human disturbance operated by the inspectors and the smoothness of the tree surface have a great

ISSN: 0010-8189

(C) CONVERTER 2020

www.converter-magazine.info 
influence on the detection process. It is difficult for the staff to keep the antenna very accurately on the same level of the cross-section of the tree trunk being tested. In the process of measurement, uniform motion cannot be achieved, and curve breakpoints appear in the feedback grayscale map of measurement results. The problem of not directly obtaining the point cloud data required by the reverse modeling of the development trend of empty rot seriously affects the accuracy of the analysis [1,2].

In order to solve the above problems existing in tree detection by TRU system and realize accurate positioning of the detection boundary of hollow rot, in-depth study takes the radar wave detection feedback pictures as the basic data and uses pixel color segmentation and 3D modeling method to obtain 3D modeling of hollow rot shape inside the trunk and reconstruction of point cloud data. The research results can be used to degenerate the two-dimensional image of the radar tree to the three-dimensional simulation in the field of empty rot, and the form of the feedback data is consistent with the human vision.

\section{The Process of Obtaining Sample Information}

In the course of the experiment, outdoor radar wave was used to detect trees and carry out laboratory data analysis after sampling. The instrument used is TRU Tree Radar detection system developed by Tree Radar Company in the United States, and the detection frequency is $900 \mathrm{MHz}$. As shown in Figure 1, the radar wave propagation path in trees and the schematic diagram of signal transmission and recovery, the detection technology of transmitted wave signal $\mathrm{T}$ and recovered wave $\mathrm{R}$ is adopted to carry out comparative inversion processing. Nondestructive testing was performed on the growth void and decayed part inside the trunk, the detection data is processed by a computer to obtain a detection result, and the feedback data of the testing results was in the form of two-dimensional gray scale map.

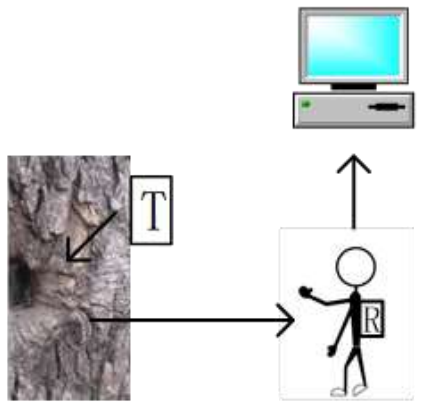

Fig 1: Signal simulation diagram of tree trunk detected by radar wave

According to the diameter of the tested specimens of trees, adjusting the operation parameters of experimental equipment for $1 \mathrm{MHz} \sim 500 \mathrm{MHz}$, high-frequency radar wave directional incoming, in the form of pulse radar wave time according to the received feedback, feedback waveform analysis processing, inferred empty inside the trunk rotting defect information such as the geometry and position, dielectric properties. The radar wave imaging data that feedback the abnormal inside the tree is in the form of a two-dimensional color gray gradient map. The boundary of the empty and rotting area in the picture is an irregular characteristic curve, which marks the incomplete surrounded area from the center of the tree to the rotting part. Since the demarcation of this area is calculated according to the difference of radar wave return signal, it is a boundary reflecting the degree of void decay [3].

Images of characteristic curve of the boundary extraction method based on OpenCV2 libraries, use of color space conversion method to extract the target pixels area, contour detection, target area to locate the empty rotting boundary curve endpoint, and draw the convex hull, complete calibration decay empty area center, morphological open operation for detecting data processing, The dryness point of the image was removed. Finally, the closed

ISSN: 0010-8189

(C) CONVERTER 2020

www.converter-magazine.info 
curve of the void decay boundary was extracted accurately.

After processing in the above way, an image with a boundary curve on a monochromatic background was obtained, and the center of the tree circle and the hollow rot area were located in the image. By connecting the characteristic curve with the center of the empty rot area, a closed fan-shaped simulation map of the empty rot area is formed. Three-dimensional morphological reconstruction was carried out according to the simulated growth defect area graphics, and the point cloud data of the hollow rot area was obtained by the inverse method. The point cloud data processing technology was used to complete the real morphological inversion of the three-dimensional structure of the hollow rot area of trees.

\section{Boundary Extraction of Experimental Process and Data Analysis Process}

According to the feedback image radar detection, this class round surround type empty boundary circle center does not coincide with the trunk and the distance between two points difference represents the internal decay empty area deviating from the tree trunk heart, therefore, the center of the calibration decay the empty area, draw the curve of fan-shaped surround, the main content of the corrosion area boundary extraction. It is an effective method to extract information according to color features.

\subsection{Boundary feature curve extraction experiment of color space conversion method}

RGB colors are hardware-oriented, the values of $R, G$ and $B$ are added together, $F=r[R]+r[G]+r[B]$, and different colors are calculated. HSV (Hue Saturation Value) color model is user-oriented. HSV color space belongs to an inverted hexagonal pyramid representation method in RGB. Hue $(\mathrm{H})$, saturation $(\mathrm{S})$ and lightness $(\mathrm{V})$ are the three parameters in the representation method. Therefore, the characteristic curve of a certain color extracted from the RGB color in the two-dimensional feedback graph needs to be transformed into the HSV color space in order to obtain a higher precision result.

\subsubsection{Color space model transformation method}

In HSV color space, the degree of hue $\mathrm{H}$ is used to represent the position of the spectral color, which is the main extracted target control parameter in the experiment. The degree of hue can be expressed by Eqs.1.

$$
\mathrm{h}=\left\{\begin{array}{ll}
0^{\circ}, & \text { if } \max ==\min \\
60^{\circ} \times \frac{\mathrm{g}-\mathrm{b}}{\max -\min }+0^{\circ}, & \text { if } \max =\mathrm{r} \text { and } \mathrm{g} \geq \mathrm{b} \\
60^{\circ} \times \frac{\mathrm{g}-\mathrm{b}}{\max -\min }+360^{\circ}, & \text { if } \max =\mathrm{r} \text { and } \mathrm{g}<\mathrm{b} ; \\
60^{\circ} \times \frac{\mathrm{b}-\mathrm{r}}{\max -\min }+120^{\circ}, & \text { if } \max =\mathrm{g} \\
60^{\circ} \times \frac{\mathrm{r}-\mathrm{g}}{\max -\min }+0^{\circ}, & \text { if } \max =\mathrm{b}
\end{array}\right\}
$$

In Equation, $r$ is equal to Red, represented the red value. $g$ is equal to Green, represented the green value. $b$ is equal to Blue, represented the blue value.

The program is written as follows. img_hsv=cv2.cvtColor (img,cv2.COLOR_BGR2HSV); plt.imshow(img_hsv) ; plt.axis('off'); plt.title('HSV') ;plt.show().

Feedback results are shown in Figure 2 after the program has been run.

ISSN: 0010-8189 
After HSV color space conversion of the original image, the image can be very intuitive to express the hue, brightness and shading of different color areas. The different colors of the curve are used to express the critical edge between the empty rot area and the healthy wood, and it is easier to track the curve color of the empty rot boundary than the RGB mode.

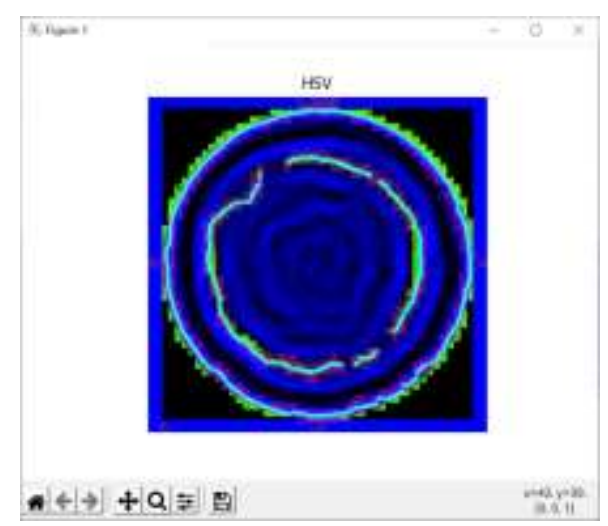

Fig 2: background highlighting using HSV format

\subsubsection{Find the target region according to the color range}

Since the value of the threshold determines the accuracy of boundary extraction, it is necessary to take the color range value closest to the target color as the middle region of the value to calibrate the values of $\mathrm{x}$ and $\mathrm{y}$, and other color regions as the values outside the target range. The background is removed to obtain the characteristic curve chart of a single color calibration.the boundary of the void decay region is demarcated as a red line, the image is binarized and the pixel values around the red region are compared. After comparison, the color space positions are obtained as two interval values [4].The value of color space given in the table 1 represents the maximum and minimum value of $\mathrm{H}, \mathrm{S}$ and $\mathrm{V}$, that is, the value range of a certain color. $\mathrm{X}$ and $\mathrm{Y}$ are the point coordinates of a certain color in the target image with close similarity, that is, the coordinates of the color in the target image being processed. Since the region of the same color in the image has multiple points, the value of coordinates is only to indicate the corresponding relationship between the color and the location of the region.

Table 1 HSV color space color range values

\begin{tabular}{|c|c|c|c|c|c|c|c|c|c|c|c|}
\hline & Black & gray & white & \multicolumn{2}{|c|}{ red } & orange & yellow & green & cyan & blue & purple \\
\hline hmin & 0 & 0 & 0 & 0 & 156 & 11 & 26 & 35 & 78 & 100 & 125 \\
\hline hmax & 180 & 180 & 180 & 10 & 180 & 25 & 34 & 77 & 99 & 124 & 155 \\
\hline smin & 0 & 0 & 0 & 43 & 43 & 43 & 43 & 43 & 43 & 43 \\
\hline smax & 255 & 43 & 30 & 255 & 255 & 255 & 255 & 255 & 255 & 255 \\
\hline vmin & 0 & 46 & 221 & 46 & 46 & 46 & 46 & 46 & 46 & 46 \\
\hline vmax & 46 & 220 & 255 & 255 & 255 & 255 & 255 & 255 & 255 & 255 \\
\hline $\mathrm{x}$ & 43 & 167 & 0 & 265 & 325 & 399 & 630 & 335 & 487 & 573 \\
\hline $\mathrm{y}$ & 30 & 161 & 0 & 178 & 150 & 129 & 398 & 173 & 182 & 203 \\
\hline
\end{tabular}

In order to evaluate the color accurately, the selected value of each coordinate in the experiment is the minimum hmin, so that the corresponding relationship between the color and the corresponding region in the picture can be calibrated uniformly. After determining the value of the coordinate, the color range can be determined according to the color values, the results are shown in Table 1.

In the experiment, the value range of $\mathrm{x}$ and $\mathrm{y}$ is obtained according to the close or similar colors as the comparison relation. Therefore, to get the accurate boundary calibration, it is necessary to carry out several experimental comparisons, and improve the threshold value of boundary color partition function to find the optimal solution. 
In order to find the location of the red region in the specific region of the image, the range of the red region is firstly calibrated. The first red region is $[0,50,50]$ and $[10,255,255]$. The second red region is $[170,50,50]$ and $[180,255,255]$. In the experiment, CV2.inrange (img_hsv, lower_red, upper_red) function is used to change the threshold value in the two red regions to 255 , and the value outside the two red regions is changed to 0 .

\subsubsection{The threshold sum method is used to demarcate the boundary}

If a curve region with width is extracted according to a particular color, the region must be of a single color, and the values of $\mathrm{H}, \mathrm{S}$, and $\mathrm{V}$ at each pixel in the region must be the same, or approximately the same within the error tolerance range.

Because there are two groups of possible values for the red region, it is necessary to deal with it in two cases. When mask=0, the parameter conditions of index np. WHERE need to be adjusted, and the position of red element should be found after feedback the color space range, and the pixel should be re-valued. When the mask $=1$, that is, the feedback value is true, the color space conversion function completes the binarization of image processing, and there is no need for the secondary conversion of index condition setting.

Reduce the error of image processing, according to the characters of gray image, the color of the narrow detection range, detection of two calibration and extraction, extraction, to directly compare the picture of the red area pixel value of HSV, and take the red areas of HSV value maximum approximation, take the red areas HSV approximate minimum value, the color of the two narrow the range interval as a reference, The deterministic HSV value is obtained by summing the two thresholds by running the function cv2.inrange.

For example, the color range of the lower mask is $[0,10]$ to obtain mask0. The conversion result is shown in Figure 3 (a). The color interval of Upper mask is [170-180], and mask1 is obtained. The conversion result is shown in Figure 3 (b). The threshold values of detection feedback were added to obtain the appropriate threshold value Mask $=\operatorname{mask} 0+\operatorname{mask} 1$.

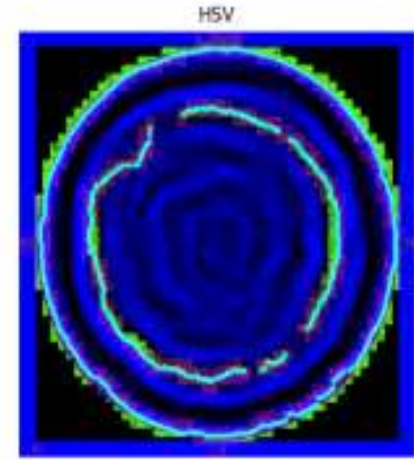

(a) Between 0 and 10

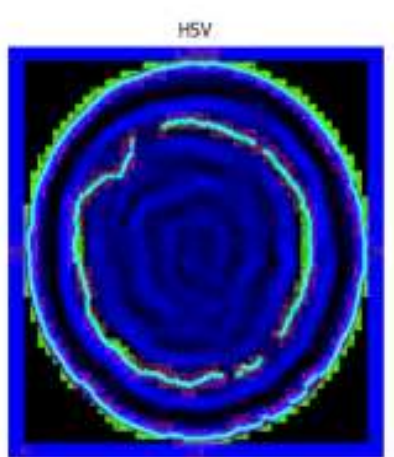

(b) Between 170 and 180

Fig 3: the result of color region transformation of characteristic curve

The experimental results show that this method can calibrate the two-dimensional void decay region boundary of the radar tree NDE feedback, and the red value of the target region is between 0-10 and 170-180. The numerical value of the calibrated boundary curve is accurate, which makes the difference between the image background and the effective boundary characteristic curve prominent, and is more convenient for the boundary extraction operation. The obtained image results were used for subsequent difference analysis. 


\subsection{Comparison of experimental results of boundary extraction with different color patterns}

In the image processing based on RGB color mode, the index condition of the image was set as np. where (mask $==0$ ), the background color of the image after extracting the boundary curve was set as white, and the color space value was $(255,255,255)$. The color of the boundary curve is red. The extraction process is described in Section 3.3.2, as shown in Figure 4. The extraction result is a red irregular curve with multiple noise points against a white background.

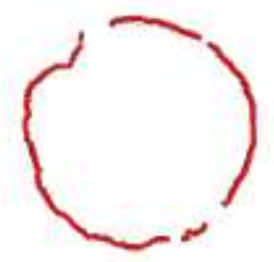

Fig 4: extraction result of red boundary curve with background gray level removed

The image with HSV color space conversion was processed, and the index condition of the image was set as np. Where (mask==0). After extracting the boundary curve, the background color of the image was black, and the color space value was $(0,0,0)$, as shown in Figure 5. The color value of the boundary curve of the extraction results is approximately blue-green, which is used to distinguish the black background. The extraction process is described in Section 3.3.2.

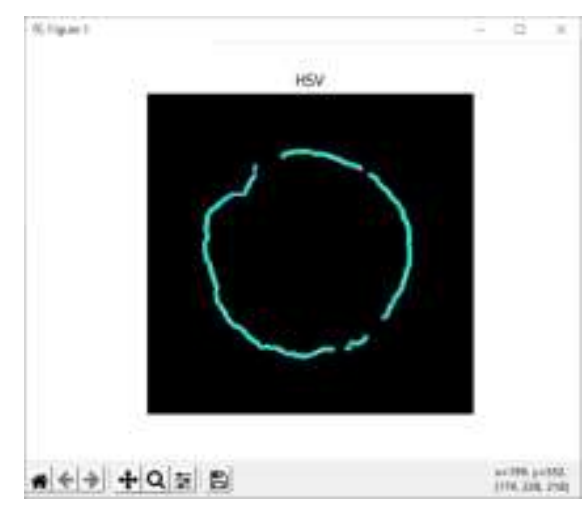

Fig 5: the results of HSV space edge extraction with background gray level removed

Because the 3D representation of the HSV model evolved from the RGB color space. Therefore, the experiment compared the boundary extraction results of RGB color mode images with those of HSV color space images. The results showed that the extracted curves under the two color modes had different widths and different noise points after binarization. Among them, the extracted images in RGB color space had more noise points, and the boundary curves had rough edges. It can not meet the requirement of smooth curve in 3D modeling.

\subsection{Reconstruction of boundary curve two-dimensional model of hollow rotting region}

In order to accurately reconstruct the two-dimensional model of hollow decay area, the white and black cross color area marked at the outermost edge of radar wave feedback picture was crossed out, that is, the data of air gap and bark thickness were not processed. In data analysis, the outer edge of the feedback picture is pre-set as a circle, and the center of the circle is directly calibrated as the center of the tree using the radar wave detector. However, the hollow rot area of the tree is deviated to a certain side of the trunk, so the tree core defined by the instrument is not the hollow rot area center. In order to get the real three-dimensional model of the growth defect of the tree, the 
hollow rot center of the defect area must be located. Locating the hollow rot center requires locating the end point of the hollow rot boundary curve first.

\subsubsection{Image denoising processing}

the binaryzation processing of dynamic adjustment threshold, Dilation, mathematical morphology processing and morphological open operation are used to remove the noise and remove white spots in the image [5].Different thresholds will be used to obtain different rendering images, compared with the processed image, according to the image segmentation effect to take the optimal threshold, the final value of parameter THRESH $=150$, the maximum gray value of MAX VAL $=255$, threshold type is THRESH_BINARY_INV, this group of parameters sampling effect is the best, the highest accuracy, the calculation formula of threshold value can be expressed by Eqs.2.

$$
\operatorname{dst}(x, y)= \begin{cases}0 & \text { if } \operatorname{src}(x, y)>\text { thresh } \\ \max v a l & \text { otherwise }\end{cases}
$$

The function automatically obtains Gmax and Gmin, which represent the maximum and minimum grayscale values of pixels obtained from the image.

The application of the opening operation method of mathematical morphology image processing is the process of Erosion and then Dilation. Operation can be expressed by Eqs.3.

$$
\mathrm{dst}=\text { open }(\operatorname{src})=\operatorname{dilate}(\operatorname{erode}(\operatorname{src}, \text { element }))
$$

The gray value of the noise that needs to be removed is a pre-estimated value. In the experiment, the bounding box algorithm with rectangular structure was adopted. The smaller the shape element of the bounding box with rectangular structure, the smaller the small white points would be removed, and the cleaner the image noise would be removed [6].The size of the elements in the rectangular bounding box is determined by parameters. In the experiment, $(5,5),(5,5)$ is used as the pre-estimated reference value.

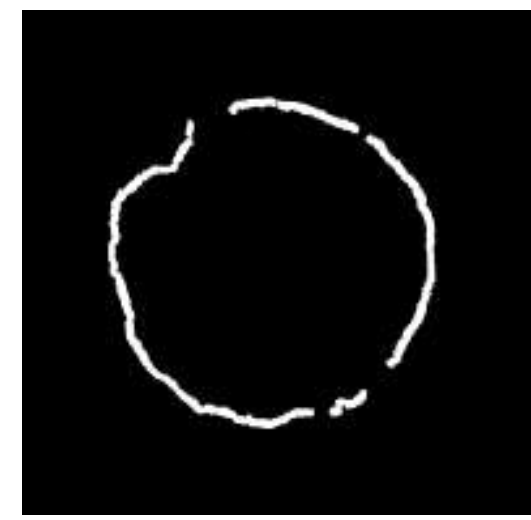

Fig 6: Screenshot after removing white spots of picture impurities

Generated boundary diagram as shown in figure 6, forecast reference identifies the noise of the small white point, some decay is empty border part effectively, so according to the target image of white spots, surrounded by secondary adjusting rectangular box size, and changing the parameters corresponding to the $(3,3),(1,1)$, effectively prevent the boundary characteristics of effective white dots in the image has been removed.

\subsubsection{Boundary extraction of empty rot region}

The Hofu circle in the original image is drawn as the target circle with the Hofu circle algorithm. The value of the

ISSN: 0010-8189 
center coordinate of the circle is. $\mathrm{x} 1=\mathrm{int}($ Circles $[0][0][0]), \mathrm{y} 1=\mathrm{int}($ Circles $[0][1])$, and the radius $\mathrm{r}=237$. The detection results are shown in figure 7 (a).

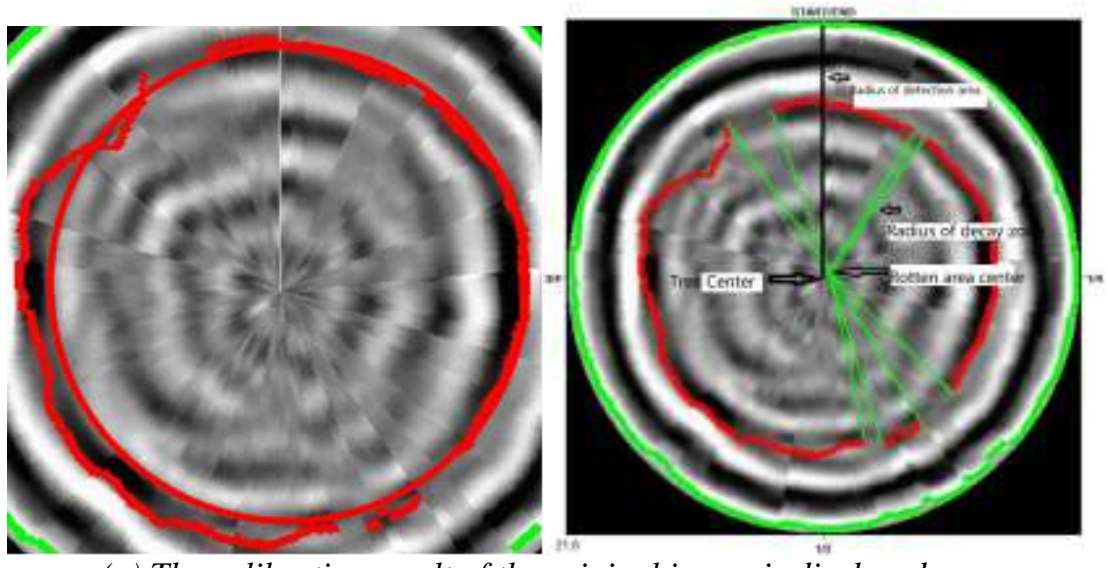

(a) The calibration result of the original image is displayed

(b) The connection result of the picture endpoint of the sampling point is identified

Fig 7: Connection result between testing center of empty rot area and end point of curve

Using G-CV2.Canny operator, noise reduction and edge detection, Circles = CV2.HoughCircles calibration, CV2.inRange function and CV2.line [7]. A line was added between the end of the boundary curve of the hollow rot area and the determined hollow rot center point, and the result was shown in Fig. 7 (b), that is, the sector edge line of the hollow rot area was demarcated, the radius of the detection area was distinguished from the radius of the hollow rot area, and the wood center was distinguished from the hollow rot center.

After finding the convex hull and drawing the convex hull (contour) on the original image, the two-dimensional boundary reconstruction results of the hollow rot region of tree growth were obtained after the completion of the operation based on Open CV2.The result is shown in Figure 8.

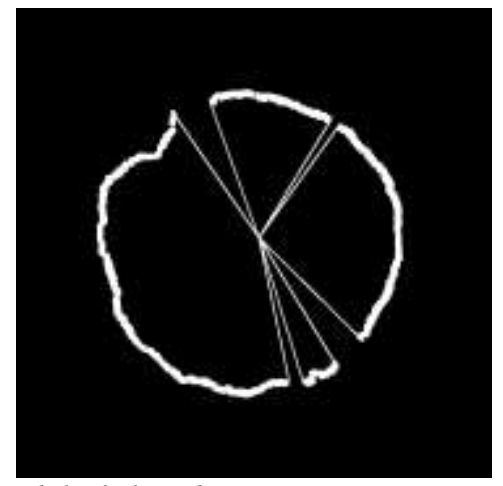

Fig 8: Boundary model of closed curve extraction in empty rot area

Staged experimental results show that the experimental method can remove the gray background of radar wave detection feedback images, and obtain a two-dimensional reconstructed data model of the complete boundary of the void decay region. Each closed sector is the actual hollow rot area, while the non-closed sector is the transition area of tree growth defects, or the loose wood area with a low degree of hollow rot.

\section{Reconstruction of 3D Point Cloud Data Based on Boundary Detection Curve}

The extraction of 2D image curve boundary based on color features into 3D modeling is to complete the high-dimensional transformation of radar wave detection data, while the point cloud data of the 3D model of empty 
rot region is the basic data form of 3D reverse modeling of multi-surface. The 3D modeling of unclear boundary based on the point cloud data registration algorithm can be used as the technical support to improve the accuracy of 3D simulation technology of radar wave nondestructive testing.

\subsection{Three-dimensional reconstruction of hollow rot region}

It is necessary to build a three-dimensional model. DWG or.dxf file form. The auxiliary software Photoshop 2017 is used to convert the image into path mode. In order to more accurately match to the desired boundary, there are two smart Pointers (handles) in each position point, and the curve drawn by the line goes to the vector shape. Using the magic wand function to eliminate the black background, export the file path to Illustrator in the create working path mode, and save the file as. AI format file, and then use Adobe Illustrator CS6 to convert. AI format file to. DWG format, so that the obtained curve is controlled by the anchor points, accuracy to meet the requirements.

Use SolidWorks to stretch the graph and set the thickness as $100 \mathrm{~mm}$. As shown in Figure 9, the thin curve on the boundary is divided into two situations. One is that the outermost thin curve is stretched into a three-dimensional entity; the other is that the boundary is directly stretched into a three-dimensional entity, and the result is stored as the extension name. SLDPRT file, and then convert the file to STL format [8,9].

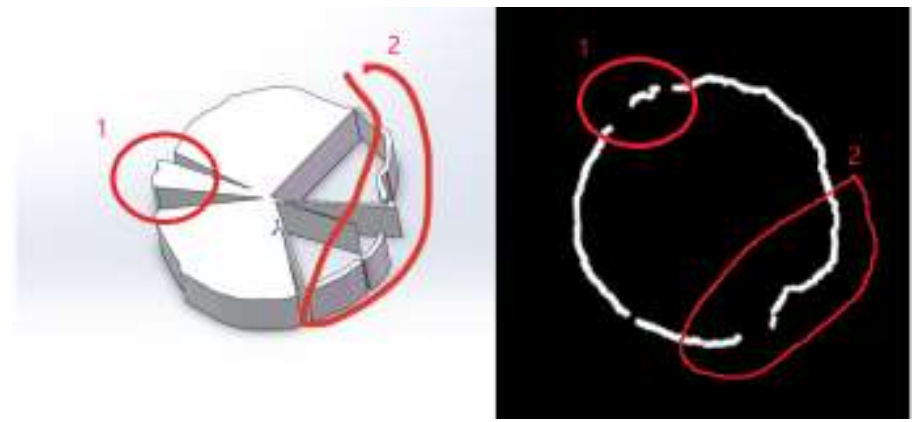

Fig 9: Correspondence between $3 D$ reconstruction model and void boundary

The correspondence between the regions labeled 1 indicates that the three-dimensional model represents a rotting region within the trunk, which is shaped like a fan connecting the boundary curve segment to the hollow rotting center. The corresponding relationship of the region labeled 2 indicates that if the void decay boundary marked on the radar detection feedback graph is coarse, the boundary is identified as a hyperbola, and the closed loop formed after 3D reconstruction is with a double boundary. If the hollow rot area where the trees grow is not completely rotted, the three-dimensional shape is an unclosed fan-shaped area. If the degree of decay gradually deepens, the fan-shaped area of the hollow rot area can also gradually grow to form a closed independent three-dimensional model area [10].

\subsection{Point cloud data reconstruction in hollow rot region}

The original STL data imported into CloudCompare, $3 \mathrm{~d}$ detection grid basic characteristics, parameters for points = 762, current HSV Angle. The parameters of the three-dimensional entity detected and reconstructed are shown in Table 2.

Table 2 Basic parameter values of triangular mesh transformation model

\begin{tabular}{|c|c|c|c|c|c|c|c|}
\hline \multirow{3}{*}{$\begin{array}{c}3 \text { d detection } \\
\text { Bounding Range } \\
\end{array}$} & \multicolumn{3}{|c|}{ coordinates } & \multirow{2}{*}{\multicolumn{2}{|c|}{ information }} & \multirow{2}{*}{$\begin{array}{l}\text { currently } \\
\text { displayed }\end{array}$} & \multirow[b]{2}{*}{ surface } \\
\hline & $X$ & $\mathrm{Y}$ & $\mathrm{Z}$ & & & & \\
\hline & 13024.4 & 13110.1 & 2540 & Object & Child Object & 3D View & \\
\hline Frame center & -4802.2 & 10019.3 & 1383.42 & 5 & 2 & 1 & 1520 \\
\hline
\end{tabular}

ISSN: 0010-8189

(C) CONVERTER 2020 
The number of triangular surfaces of the target 3D entity detected is 1520 . In the 3D view with 5 objects and 2 sub-objects, the frame range that can be operated is $\mathrm{X}=13024.4, \mathrm{Y}=13110.1, \mathrm{Z}=2540$. The center coordinates of the border are $(-4802.2,10019.3,1383.42)$.

After 3D wireframe processing, the wireframe division of the three-dimensional model of the void and rot area is obtained, as shown in Figure 10. The selection grid in Fig. 11 (a) is marked with normal lines, which are the hidden black lines in the figure. Fig. 11 (b) Select the grid normals and demarcate them as non-hidden lines, and the number and shape of wireframes are different.

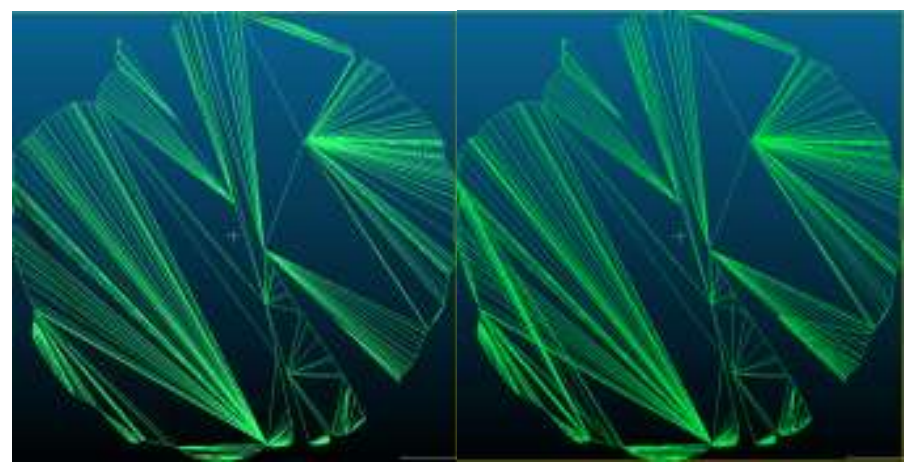

(a) Select the grid normal to be empt $\quad$ (b) the grid normal is the true value Fig10: Screenshot after 3D wireframe processing

To carry out point cloud data matching, non-empty data should be returned, that is, the normal vector exists. Therefore, the grid normal is selected as true value. In this experiment, the mesh subdivision parameter of 1.00000100 was used as the parameter to find the optimal value. The screenshot of the generated point cloud data is shown in Figure 11.

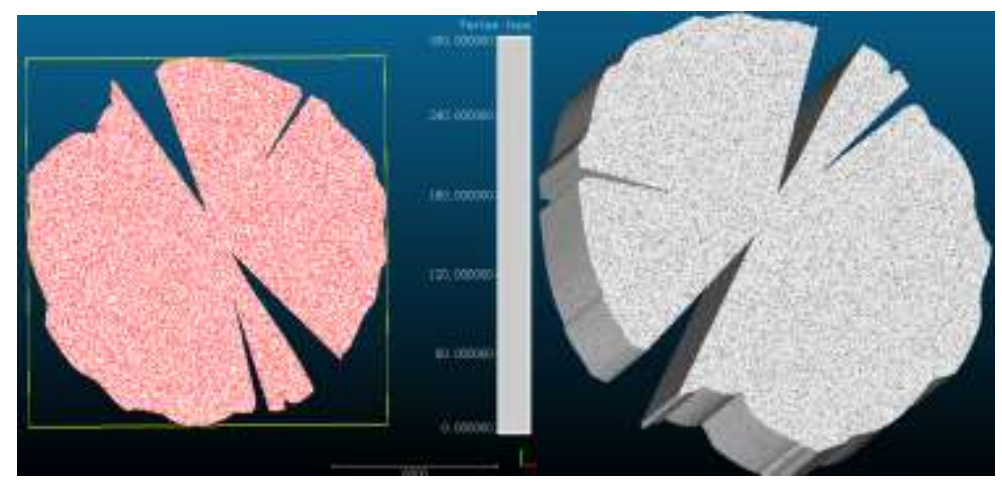

Fig 11: point cloud reconstruction model in empty rot area

The point cloud data of empty rot region is finally obtained through the conversion of 3D model data format. Set the number of generation point clouds as 2 million, and the actual number of generation point clouds is 1999993.

\section{Experimental Error Analysis}

The parameters of the original model and the amount of triangular mesh data determine the performance index of point cloud data processing. When these parameters are imported into the format converter of point cloud data processing, there will be some changes, such as the loss of point cloud data and the number of triangular mesh partitioning, etc. These reasons cause experimental errors. Need to adjust the parameters and scaling.Specific data are shown in Table 3.

ISSN: 0010-8189 
Table 3 Scaling parameter settings of imported raw data

\begin{tabular}{|c|c|c|c|}
\hline coordinates & $\mathrm{X}$ & $\mathrm{Y}$ & $\mathrm{Z}$ \\
\hline original value & 14833.599609 & 5778.500000 & 113.417953 \\
\hline scaling parameter & -14800.00 & 0 & 0 \\
\hline Application of numerical & 33.5996 & 5778.5000 & 113.4180 \\
\hline
\end{tabular}

Since the original coordinate value in the import process was too large, the method of estimation was adopted in the import. The estimated value of $\mathrm{X}$ was reduced to -14800 , and the error value was 1.00 , after the above imported data is estimated and reduced, the basic parameters of the measured STL grid model are shown in Table 4.

Table 4 Measurements Measured surface area and volume values of the mesh

\begin{tabular}{|l|l|l|l|}
\hline Mapping operations & parameter types & $\begin{array}{l}\text { Quantitative value (scientific } \\
\text { method of enumeration) }\end{array}$ & Sampling time \\
\hline $\begin{array}{l}\text { The total surface number of mesh } \\
\text { transformation }\end{array}$ & Square unit & $4.35678 \mathrm{E}+8$ & 12.49 .06 \\
\hline Mean triangular surface area & Square unit & 286630 & 12.49 .06 \\
\hline Mesh transformation volume & Square unit & $3.08097 \mathrm{E}+11$ & 13.03 .20 \\
\hline
\end{tabular}

The total surface area of the measured mesh model is 435678000 square units, and the average surface area of the measured triangle pieces is 28663 square units, and the ratio of the two is 1520.0013956 . The measured volume of the closed mesh was 308,097,000,000 cubic units. The number of faces counted in Table 2 is 1250 . These two data are approximately equal, so that the error of imported data is within the allowable range set by the experiment.

\section{Conclusions}

In this study, the radar wave was used for the nondestructive testing of tree growth defects, which realized the 2-D to 3-D mapping of the testing feedback data and generated point cloud data, which provided technical support for the application of point cloud data to simulate the degree of hollow decay in the nondestructive testing of radar wave. The experimental conclusions are as follows:

1. The boundary extraction methods of different color Spaces are compared. Finally, HSV color space data conversion is used to set the conversion threshold according to the color range region close to the target pixel value; after summing the thresholds, the boundary extraction is realized by combining the method of 3D array to store the image information.

2. The mathematical morphology image processing method is used to connect the center of the empty rot area and the end point of the boundary curve to realize the two-dimensional extraction of the boundary of the empty rot area to the three-dimensional reverse modeling.

3. Completed the 3D feature calibration and recognition based on multi-software data format transformation, completed the 3D wireframe processing, calculated the triangular surface data, and obtained the accurate 3D point cloud reconstruction laboratory conclusion.

4. Optimize the subdivision mesh parameters in point cloud data reconstruction through experimental error analysis, reset the error value of scaling parameters, improve accuracy and reduce error.

Core technology, using the theory of reverse engineering in this method can quickly detect the trees $3 \mathrm{~d}$ status, the internal defects detection feedback data in the form of a $3 \mathrm{~d}$ model accurately reproduce the trees empty corruption 
case, and get a $3 \mathrm{~d}$ point cloud data, complete the testing feedback model and the processing result of human visual consistency, improve the radar wave nondestructive testing data trees secondary analysis ability, Reduced technical barriers for non-specialists to use radar waves for nondestructive testing of trees.

\section{Acknowledgments}

This research was supported by The Fundamental Research Funds in Heilongjiang Provincial Universities (135409225, 135409606) and scientific research projects of basic scientific research business expenses in Heilongjiang Provincial Colleges and Universities (135409102).

\section{References}

[1] X.Y. Xiao, J. Wen, Z.L. Xiao, W.L. Li, H.J. Zhang, "Detection and recognition of tree structure internal defects based on radar," Forestry science, vol. 54, no. 05, pp. 127-134, 2018.

[2] M.X. Gan, T. Sun, Y.X. Kang, X.D. Liu, X.J. Li, "Examination of the Trunk Cvity and Thick Root Distribution of Acient Platycladus orientalis in the Tomb of Yellow Emperor By Ground Penetrating Radar Technology," Journal of Northwest Forestry College, vol. 31, no. 04, pp. 182-187+194, 2016.

[3] H.L. Xu, "Research on Feature Points Detection and Matching Algorithm for Tree Images," Master's dissertation, Northeast Forestry University, 2017.

[4] X.S. Cai, J.B. Dai, X.N. Li, "Median Filter Method and Average Value Filter Method in the Application of Bar Code Denoising," Journal of Changchun Normal University(Natural Science), vol. 27,no. 08, pp. 40-42, 2008.

[5] Y.J. Liu, S.M. Zhang, J. Feng, "Binary image dilation based on finite automata," Journal of Northwest Normal University(Natural Science), vol. 48, no. 03, pp. 48-51+59, 2012.

[6] J.J. Chen, J.F. Xu, "Application of new technology and new equipment in the general survey of basic information of underground pipelines in Beijing," Urban survey, no. 01, pp. 122-127, 2018.

[7] X.P. Wang, "Research on Image Processing Filtering Algorithm Based on OpenCV," Modern industrial economy and informatization, vol. 08, no. 06, pp. 61-62, 2018.

[8] N. Li, G.L. Duan, H.J. Xu, "Research on way of data exchange between Pro/E and adams," Mechanical design, vol. 29, no. 09, pp. 36-40, 2012.

[9] A. Mohammad, G. Akbar, A. Haluk, "Ebrahim Asghari-KaljahiDevelopment of a 2D and 3D computational algorithm for discontinuity structural geometry identification by artificial intelligence based on image processing techniques," Bulletin of Engineering Geology and the Environment, vol. 78, no. 05, pp. 3371-3383, 2019.

[10] Y.P. Chen, T. Gao, D.S. Li, W.J. Guo, "Preliminary study on radar detection and imaging of cavities and cracks of Pinus massoniana,” Journal of Beijing Forestry University, vol. 39, no. 03, pp. 112-118, 2017. 